\title{
EL GÉNERO EURYCOTIS \\ (DICTYOPTERA: BLATTARIA: BLATTIDAE: POLYZOSTERIINAE) \\ 1. ESPECIES DE LA HISPANIOLA. SEGUNDA PARTE: SIETE ESPECIES NUEVAS
}

\section{Esteban Gutiérrez}

Museo Nacional de Historia Natural, Obispo No. 61, Esq. Oficios, Plaza de Armas, La Habana Vieja 10100, La Habana, Cuba. esteban@mnhnc.inf.cu; byrsotria2002@mnhnc.yahoo.com

\section{RESUMEN}

Se describen siete especies nuevas del género de cucarachas Eurycotis Stål para la República Dominicana (Hispaniola). Se brindan diagnosis diferenciales, ilustraciones y comentarios para los táxones nuevos, y fotografías de los ejemplares tipos macho y hembra de E. tibialis Hebard, 1916.

Palabras clave: Dictyoptera, Blattaria, Blattidae, Polyzosteriinae, cucaracha, especies nuevas, Eurycotis, Hispaniola, República Dominicana, distribución.

Title: The cockroach genus Eurycotis (Dictyoptera: Blattaria: Blattidae: Polyzosteriinae). 1. Species from Hispaniola. Second Part: seven new species.

\section{ABSTRACT}

Seven new species of the cockroach genus Eurycotis Stål are described from the Dominican Republic (Hispaniola Island). Differential diagnoses, illustrations and comments are given for the new taxa, as well as pictures of the male and female type specimens of E. tibialis Hebard, 1916.

Keywords: Dictyoptera, Blattaria, Blattidae, Polyzosteriinae, cockroach, new species, Eurycotis, Hispaniola, Dominican Republic, distribution.

\section{INTRODUCCIÓN}

En la primera parte del estudio sobre el género Eurycotis Stål en la Hispaniola (Gutiérrez, 2013), se describieron seis especies nuevas de estas cucarachas para la República Dominicana (Hispaniola), brindándose diagnosis diferenciales, ilustraciones y comentarios para los táxones nuevos y las dos especies previamente conocidas de la isla. De esta manera, se incrementó a ocho especies el conocimiento sobre la diversidad del género Eurycotis en la Hispaniola y a 52 en América.

En este segundo trabajo se adicionan siete especies nuevas para la República Dominicana, elevándose a 15 la diversidad del grupo en este país y a 59 en América. Debido a la alta especiación del género encontrada durante el presente trabajo, se propone continuar profundizando en el estudio de las especies de la Hispaniola, con miras a publicar sus resultados en una tercera parte. 


\section{OBJETIVOS}

- Describir siete especies nuevas del género Eurycotis.

- Confeccionar diagnosis e ilustraciones para cada una de las especies descritas del género en el presente estudio.

\section{MATERIALES Y MÉTODOS}

El material estudiado proviene del Museo Nacional de Historia Natural, La Habana, Cuba (MNHNCu); The Academy of Natural Sciences of Philadelphia (ANSP), Pensilvania; National Museum of Natural History, Smithsonian Institution (NMNH), Washington D.C; Florida State Collection of Arthropods (FSCA), Gainesville, FL, y del Museo Nacional de Historia Natural "Prof. Eugenio de Jesús Marcano", República Dominicana (MNHNSD).

La técnica empleada para la disección y estudio de los genitales del macho, las mediciones de los ejemplares de los táxones nuevos, la confección de láminas y los materiales utilizados, como se explica en Gutiérrez (2013).

Se siguió el sistema de clasificación de McKittrick (1964) y Roth (2003). Abreviaturas empleadas: $\mathrm{M}=$ macho, $\mathrm{H}=$ hembra, $\mathrm{y} \mathrm{N}=$ tamaño de la muestra. Todas las medidas en milímetros.

\section{RESULTADOS}

\section{TAXONOMÍA}

\section{Género Eurycotis Stål 1874}

Especie Tipo: Periplaneta mysteca Saussure, 1862 = Polyzosteria rufovittata Brunner, por Monotipia.

\section{Eurycotis tibialis Hebard \\ Figura 1, A-K}

Eurycotis tibialis Hebard, 1916: 264.

Ilustraciones en: Hebard, 1916, Lám. 14, Fig. 1 y figura con texto; Gurney, 1942, Lám. 1, Fig. 4, Lám. 2, Figs. 10, 20; Gutiérrez, 2013, Fig. 1, A-D.

Comentario. Gutiérrez (2013) acerca de Eurycotis tibialis comentó: “...debido a la inseguridad de la procedencia exacta del holotipo ("San Francisco Mountains, San Domingo") y a la amplia distribución de los ejemplares adicionales estudiados en la República Dominicana... se requiere el estudio de los genitales del holotipo... para realizar estudios comparativos que permitan definir los posibles táxones nuevos relacionados con esta especie". El holotipo macho está depositado en el NMNH de Washington. Debido a lo destructivo que resulta para el ejemplar la disección de los genitales, y con la finalidad de continuar nuestros estudios comparativos, se confeccionó una lámina con fotografías del holotipo y el alotipo de este taxon, gentilmente enviadas por colegas del Smithsonian. A continuación se describe una especie nueva emparentada con E. tibialis.

\section{Eurycotis samana sp. nov.}

Figura 2, A-K

Diagnosis. Eurycotis samana sp. nov., comparte coloración (unicolor), tegminas separadas, tibia III ensanchada y modificada con E. tibialis (República Dominicana, Hispaniola) y E. biolleyi 
(Costa Rica y Panamá). La especie nueva difiere de E. biolleyi en la forma de las tegminas (margen interno convexo en E. biolleyi) y en la depresión ovalada de la tibia III (Fig. 2F) (surco longitudinal en E. biolleyi). Eurycotis samana sp. nov., es mucho más pequeña y delgada que E. tibialis (en el macho) (forma más grande y robusta en tibialis), difiere en su menor longitud del cuerpo (samana=21.93, Fig. 2, A-B; tibialis=27.5, Fig. 1, A-B), longitud y amplitud del pronoto $($ samana $=7.14 \times 9.69 ;$ tibialis $=9.4 \times 13.3)$, longitud y amplitud de la tegmina (samana=3.91 $\mathrm{x}$ 2.55; tibialis $=6.8 \times 3.6$ ) la cual es menos ancha en su base que tibialis en relación a su amplitud apical (samana, Fig. 2E; tibialis, Fig. 1D), longitud de la tibia caudal y artejos tarsales caudales ( amana $=7.48 \times 6.46$; tibialis=11.2 $\times$ 8.9), placa supra-anal del macho con invaginación media en forma de "V invertida abierta" que divide su margen caudal en dos líneas rectas (Fig. 2H) ("V invertida cerrada" con margen caudal dividido en dos líneas redondeadas en tibialis, Fig. 1G), y en tener el margen caudal de la placa subgenital menos cóncavo (Fig. 2I) que en tibialis (Fig. 1H).

Diagnosis. Eurycotis samana sp. nov., shares coloration (unicolor), separate tegmina, broadened and modified tibia III, with E. tibialis (Dominican Republic, Hispaniola) and E. biolleyi (Costa Rica and Panama). The new species differs from $E$. biolleyi in the shape of the tegmina (convex inner margin in E. biolleyi), and in the ovate depression on the caudal face of tibia III (Fig. 2F) (longitudinal sulci in E. biolleyi). Eurycotis samana sp. nov., is smaller and slender than E. tibialis (in male) (larger and robust shape in tibialis), it differs in its smaller body length (samana=21.93, Fig. 2, A-B; tibialis=27.5, Fig. 1, A-B), pronotum length and width (samana=7.14 x 9.69; tibialis $=9.4 \times 13.3)$, tegmen length and width (samana $=3.91 \times 2.55$; tibialis $=6.8 \times 3.6)$ which is less wide in its base than tibialis in relation with its apical width (samana, Fig. 2E; tibialis, Fig. 1D), length of caudal tibia and tarsal caudal joints (samana=7.48 x 6.46; tibialis $=11.2 \mathrm{x}$ 8.9), male supra-anal plate with medial invagination "inverse V-shaped, opened" which splits its caudal margin in two straight lines (Fig. 2H) ("inverse V-shaped, closed" with its caudal margin divided in two rounded lines in tibialis, Fig. 1G), and in having the subgenital plate caudal margin less concave (Fig. 2I) than in tibialis (Fig. 1H).

Descripción. Macho holotipo: superficie dorsal del cuerpo lisa, lustrosa, cubierta por puntuaciones muy finas, separadas, ligeramente rugosa a nivel de la placa supra-anal (Fig. 2, A, H). Cabeza ligeramente expuesta, distancia entre las bases de las antenas ligeramente menor que el espacio interocular, manchas ocelares tenues (Fig. 2C). Pronoto con los márgenes ántero-laterales y ángulos postero-laterales redondeados, el margen caudal recto, (Fig. 2D). Tegminas laterales, ligeramente más anchas en la base que en el ápice; margen interno recto a ligeramente convexo; márgenes látero-caudales ligeramente redondeados, sobrepasan ligeramente el margen posterior del mesonoto (Fig. 2E). Alas posteriores ausentes. Fémur I con margen anterior con hilera de espinas robustas, que terminan en tres espinas apicales más largas y engrosadas (Tipo $\mathrm{A}_{3}$ ); fémures II y III con espina genicular; tibia posterior ensanchada, no inflada, con modificación en forma de depresión ovalada en su cara caudal (Fig. 2F), y en forma de depresión ligeramente elíptica en su cara cefálica (Fig. 2G); pulvilos sobre los cuatro tarsómeros proximales; uñas tarsales simétricas, simples, arolio desarrollado. Abdomen con ángulos caudo-laterales del cuarto al séptimo terguito abdominal ligeramente proyectados en forma de espinas, finos, puntiagudos, sin lamelas. Placa supra-anal transversal; margen caudal con invaginación media en forma de "V" invertida abierta, cubierta por cerdas; márgenes laterales sinuosos y ángulos látero-caudales redondeados; no sobrepasa la longitud de los cercos (Fig. 2H). Placa subgenital con margen posterior ligeramente cóncavo; estilos simples, ligeramente recurvados en su porción media (Fig. 2I). Genitales con el esclerito R2 del falómero derecho como se muestra en la Fig. 2K. Color: superficie dorsal unicolor, negro con viso rojizo. Superficie ventral oscura que contrasta con pálido de las manchas ocelares y pulvilos, y con naranja-pálido en márgenes de coxas y 3/4 partes de los tarsómeros apicales. 

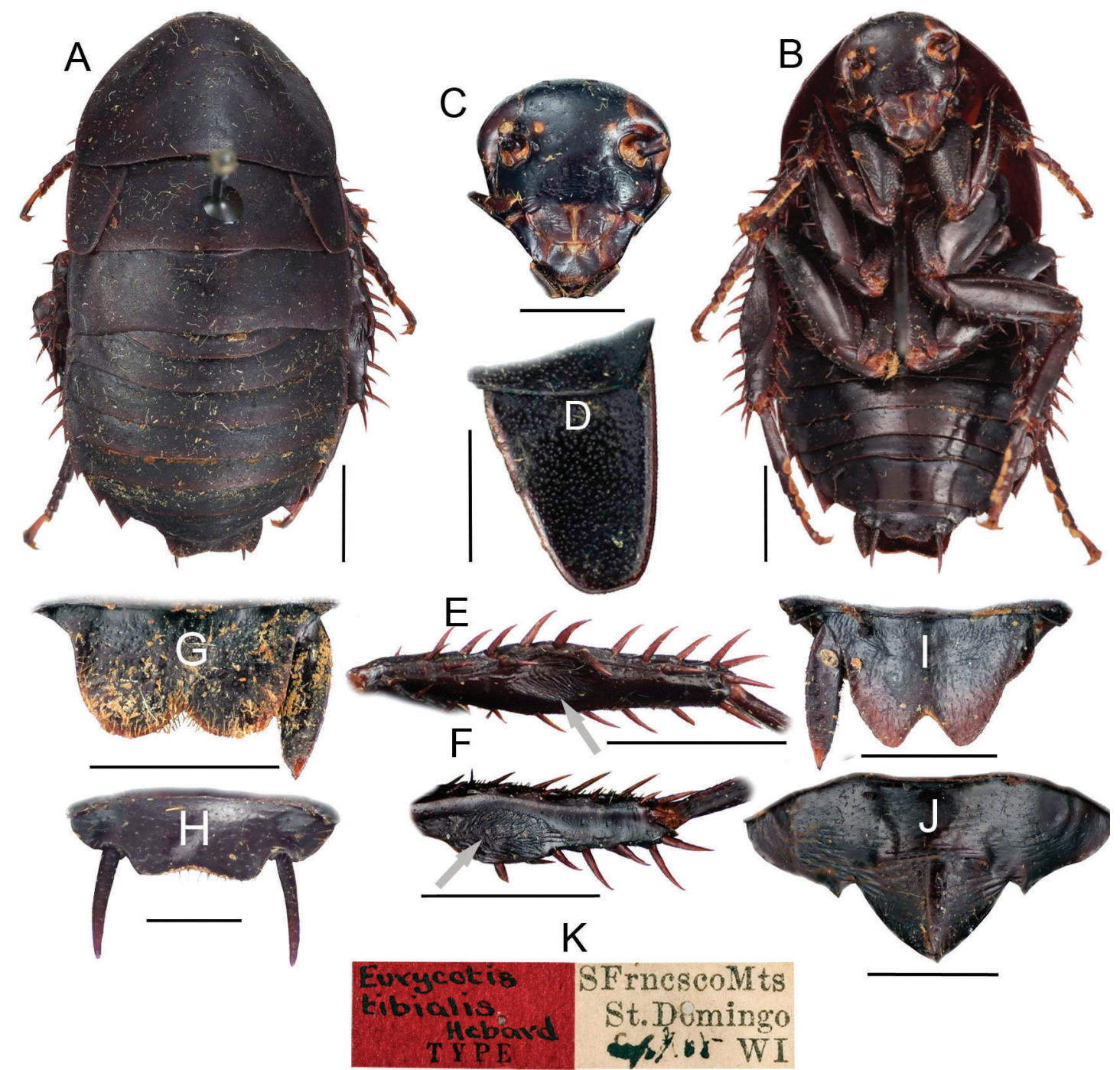

Figura 1, A-K. Eurycotis tibialis Hebard, 1916. A-H, holotipo macho del NMNH. A, aspecto dorsal del cuerpo. B, cuerpo (ventral). C, cabeza (frontal). D, tegmina derecha (dorsal). E, tibia III derecha (cara caudal), la flecha indica concavidad ovalada. F, tibia III derecha (cara cefálica), la flecha indica área rugosa elíptica. G, placa supra-anal y cerco derecho (dorsal). H, placa subgenital y estilos (ventral). I-J, alotipo hembra. I, placa supra-anal y cerco izquierdo (dorsal). J, placa subgenital (ventral). K, etiquetas del holotipo. Escalas: $\mathrm{A}-\mathrm{B}=6.8 \mathrm{~mm}, \mathrm{C}=3.7 \mathrm{~mm}, \mathrm{D}=3.4 \mathrm{~mm}, \mathrm{E}-\mathrm{F}=5.6 \mathrm{~mm}, \mathrm{G}=2.5 \mathrm{~mm}, \mathrm{H}=1.25 \mathrm{~mm}, \mathrm{I}-\mathrm{J}=2.5 \mathrm{~mm}$. 


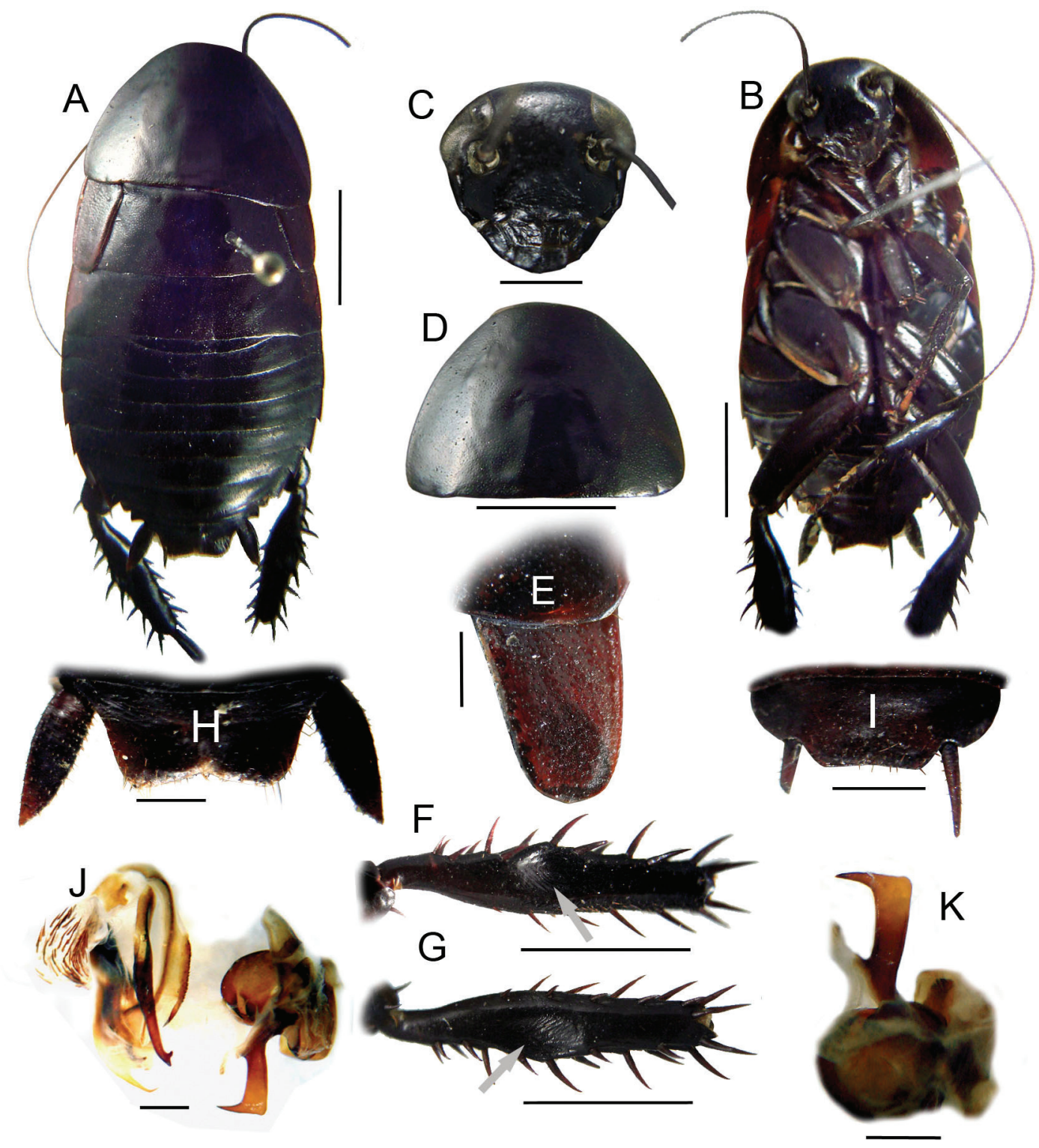

Figura 2, A-K. Eurycotis samana sp. nov., holotipo macho del NMNH. A, aspecto dorsal del cuerpo. B, cuerpo (ventral). C, cabeza (frontal). D, pronoto (dorsal). E, tegmina derecha (dorsal). F, tibia III derecha (cara caudal), la flecha indica concavidad ovalada. $\mathrm{G}$, tibia III derecha (cara cefálica), la flecha indica área rugosa ligeramente elíptica. H, placa supra-anal y cercos (dorsal). I, placa subgenital y estilos (ventral). J, estructuras genitales (dorsal). K, esclerito R2 del falómero derecho (ventral). Escalas: A-B=4.8, $\mathrm{C}=2.7, \mathrm{D}=3.57 \mathrm{~mm}, \mathrm{E}=1.27 \mathrm{~mm}, \mathrm{~F}-\mathrm{G}=3.74 \mathrm{~mm}, \mathrm{H}=1.3 \mathrm{~mm}, \mathrm{I}=1.7 \mathrm{~mm}, \mathrm{~J}-\mathrm{K}=1 \mathrm{~mm}$. 
Etimología. El nombre propuesto alude a la península de Samaná y a la provincia del mismo nombre, donde se encuentra la localidad tipo de la especie.

Medidas (mm). Macho: longitud del cuerpo, 21.93, longitud del pronoto x amplitud, 7.14 x 9.69, longitud de la tegmina $\mathrm{x}$ amplitud, $3.91 \times 2.55$, longitud de la tibia, 7.48, longitud de los tarsos, 6.46. Nota: macho $\mathrm{N}=1$.

Tipos. Holotipo. M, REPÚBLICA DOMINICANA, RD-179, La Laguna, N E1 Valle, provincia Samaná, 28-29-XI-2003, 54 m, 19 $15.007^{\prime}$ N, 69 18.471’W, D. Pérez, R. Bastardo, A. Francisco; depositado en el NMNH.

Comentario. Eurycotis samana sp. nov. solo se conoce de su localidad tipo. La especie es conocida hasta la fecha del único ejemplar macho aquí estudiado. Con recolectas futuras se definirá la distribución de la especie en la isla.

Eurycotis adamesi sp. nov.

Figuras 3, A-L; 4, A-B

Eurycotis sp. “unknown species 4", en: Gutiérrez y Pérez-Gelabert (2000), pág. 427. Eurycotis sp. 2, en: Perez-Gelabert (2008), pág. 143.

Diagnosis. Eurycotis adamesi sp. nov., comparte el carácter tibia III ensanchada e hinchada solo con E. rothi y E. neiba sp. nov. de República Dominicana. De E. rothi y E. neiba sp. nov., se diferencia en presentar la tibia III menos ensanchada e hinchada (Fig. 3, F-H) (E. rothi, Fig. 7, E-G en Gutiérrez, 2013; E. neiba sp. nov., Fig. 5, F-H). De E. rothi difiere además, en su menor talla (E. adamesi sp. nov., M: 17.3 y H: 18.54 ; E. rothi, M: 19.8 y H: 19.4 ) y menor longitud x amplitud de la tegmina (E. adamesi sp. nov., M: 3.4 x 1.92 y H: 3.49 x 2; E. rothi, M: 4 x 2.3 y H: 4 x 2.26). De ambas especies difiere en la placa supra-anal (Fig. 3I) (E. rothi, Fig. $7 \mathrm{H}$ en Gutiérrez, 2013; E. neiba sp. nov., Fig. 5I), así como en la forma del esclerito R2 del falómero derecho del macho (Fig. 3L) (E. rothi, Fig. 7K en Gutiérrez, 2013; E. neiba sp. nov., Fig. 5L).

Diagnosis. Eurycotis adamesi sp. nov., shares the character widened and inflated tibia III only with $E$. rothi and E. neiba sp. nov. from Dominican Republic. From E. rothi and E. neiba sp. nov., it differs in having tibia III less widened and inflated (Fig. 3, F-H) (E. rothi, Fig. 7, E-G in Gutiérrez, 2013; E. neiba sp. nov., Fig. 5, F-H). From E. rothi it also differs in its smaller size (E. adamesi sp. nov., M: 17.3 and H: 18.54; E. rothi, M: 19.8 and H: 19.4) and smaller tegmen length $\mathrm{x}$ width (E. adamesi sp. nov., M: 3.4 x 1.92 and $\mathrm{H}: 3.49$ x 2; E. rothi, M: 4 x 2.3 and $\mathrm{H}: 4 \times 2.26$ ). From both species it differs in supra-anal shape (Fig. 3I) (E. rothi, Fig. 7H in Gutiérrez, 2013; E. neiba sp. nov., Fig. 5I), as well as in the shape of sclerite R2 of the male right phallomere (Fig. 3L) (E. rothi, Fig. 7K in Gutiérrez, 2013; E. neiba sp. nov., Fig. 5L).

Descripción. Macho holotipo: $17.3 \mathrm{~mm}$. Superficie dorsal del cuerpo lisa, lustrosa, con puntuaciones muy finas perceptibles con mucho aumento. Cabeza ligeramente expuesta; distancia entre las bases de las antenas ligeramente menor que el espacio interocular (Fig. 3C). Pronoto subparabólico con el margen caudal truncado (Fig. 3D). Tegminas laterales, escamiformes, más anchas en la base, se estrechan hacia el ápice, margen apical ligeramente recto, no sobrepasan el margen posterior del mesonoto (Fig. 3, A y E). Alas posteriores ausentes. Fémur I con margen anterior con hilera de espinas robustas, que terminan en tres espinas apicales más largas y engrosadas (Tipo $\mathrm{A}_{3}$ ); fémures II y III con espina genicular; tibia III (caudal) con sus caras caudal y cefálica ligeramente ensanchadas (Fig. 3, F y H), y ligeramente infladas (se observa en el margen dorsal, Fig. 3G) en su porción media, sin modificación como en E. tibialis 

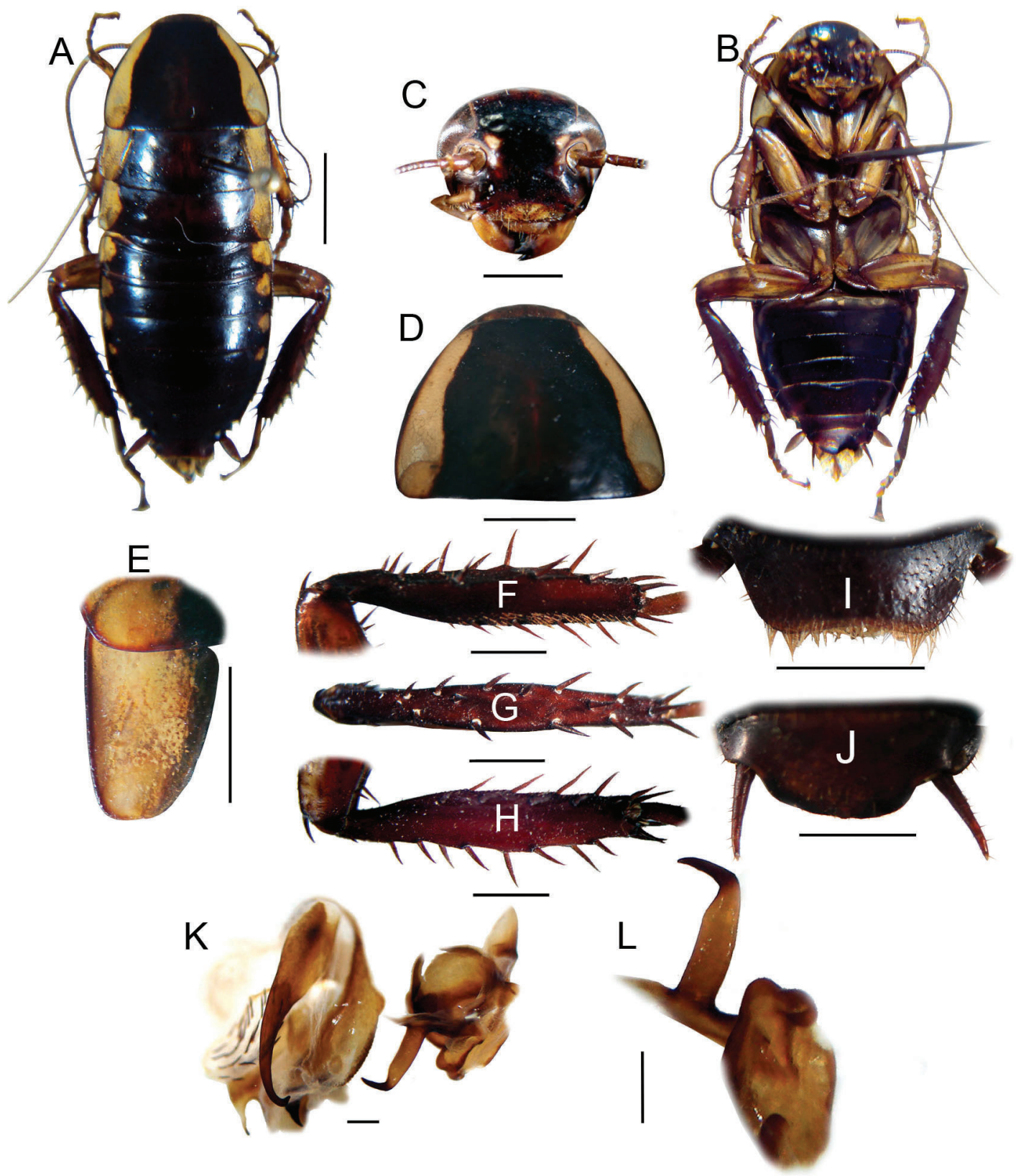

Figura 3, A-L. Eurycotis adamesi sp. nov., holotipo macho del MNHNCu. A, aspecto dorsal del cuerpo. B, cuerpo (ventral). C, cabeza (frontal). D, pronoto (dorsal). E, tegmina izquierda (dorsal). F, tibia III derecha (cara caudal). G, ídem anterior (margen dorsal). H, tibia III derecha (cara cefálica). I, placa supra-anal (dorsal). J, placa subgenital y estilos (ventral). K, estructuras genitales (dorsal). L, esclerito R2 del falómero derecho (ventral). Escalas: A-B=5.5 mm, C=1.8 mm, D=2.7 mm, E=1.9 mm, F-H=1.75 mm, $\mathrm{I}=2.1 \mathrm{~mm}, \mathrm{~J}=1.1 \mathrm{~mm}, \mathrm{~K}-\mathrm{L}=0.4 \mathrm{~mm}$. 

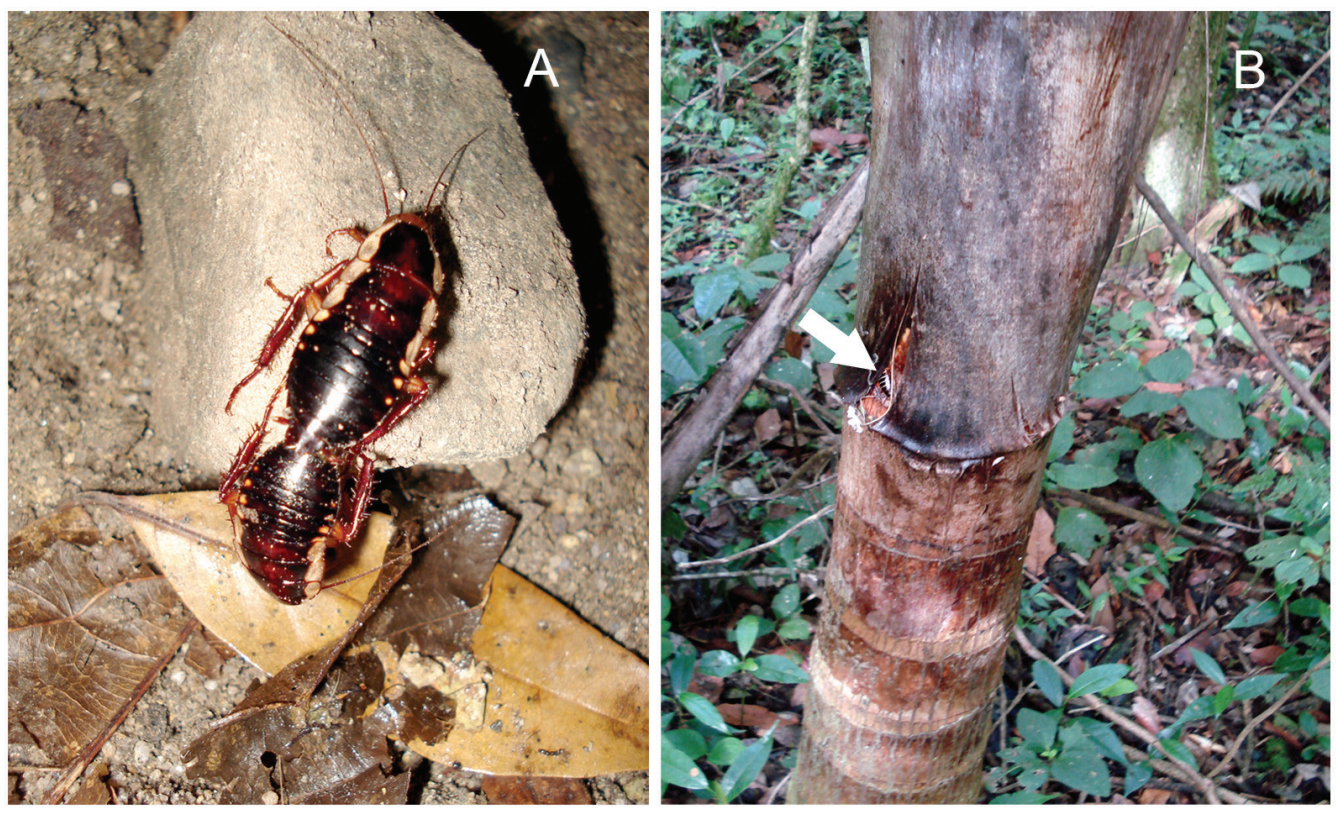

Figura 4, A-B. Refugios de Eurycotis adamesi sp. nov. en el Parque Nacional Armando Bermúdez. A, pareja sorprendida en cópula al ser recolectada bajo piedras y hojarasca, obsérvese su conducta de "congelación", inmóviles para despistar a los depredadores. $\mathrm{B}$, la flecha indica a un individuo refugiado bajo una vaina de palma manacla en pie.

(Fig. 1, E-F); pulvilos sobre los cuatro tarsómeros proximales; uñas tarsales simétricas, simples, arolio desarrollado. Abdomen con ángulos caudo-laterales del séptimo terguito abdominal simples (no proyectados), sin lamelas. Placa supra-anal con el margen caudal cóncavo, márgenes laterales sinuosos; ángulos látero-caudales redondeados (Fig. 3I); cercos puntiagudos sobrepasan placa supra-anal. Placa subgenital ligeramente asimétrica, margen caudal convexo, proyectado entre los estilos; estilos simples, largos, ligeramente recurvados y afinados en el ápice (Fig. 3J). Genitales con el esclerito R2 del falómero derecho como en la Fig. 3L. Color: superficie dorsal (Fig. 3A) bicolor; negra (con viso marrón-rojizo al estereoscopio) que contrasta con dos bandas anchas amarillo-pálido a naranja (blanco-pálido en vida a nivel del pro-, mesoy metanoto, Fig. 4A) que corren a ambos lados del cuerpo y comienzan a nivel de los ángulos ántero-laterales del pronoto hasta los ángulos látero-caudales, continúa a nivel de las tegminas (bicolores, mayormente amarillo naranja pálido excepto banda oscura en el margen interno, Fig. $3 \mathrm{E}$ ), el metanoto y se hacen intermitentes y más estrechas en los terguitos 2-5 (carácter variable en diversas poblaciones estudiadas de la especie, desde 4 a 2 manchas tergales). Superficie ventral (Fig. 3B): cabeza negra con amarillo naranja pálido en clípeo, labro, palpos, 3/4 partes de las mandíbulas; marcas ocelares blanco pálido contrastantes (Fig. 3C). Amarillo con viso rojizo opaco en coxas, trocánteres, fémures, tibias I y II; castaño rojizo en tibias III; amarillo castaño en tarsos, blanco pálido en pulvilos y abdomen castaño-oscuro a negro. Hembra: difiere del macho en su mayor talla, en las placas: supra-anal (subtriangular proyectata con invaginación apical), y subgenital (bilobular) características de las hembras del género.

Etimología. Dedicada a Germán de Jesús Adames, supervisor de la División Norte del Parque Nacional Armando Bermúdez, por su valiosa asistencia en el trabajo de campo, quien con su experiencia y conocimiento del área, propició la recolecta y documentación del taxon aquí descrito. 
Medidas (mm). Hembra entre paréntesis, media entre corchetes: longitud del cuerpo, 14.4519.72 [17.3] (16.15-21.42 [18.54]), longitud del pronoto $x$ amplitud, 5.1-6.12 [5.58] x 5.95-7.82 [7.03] (5.1-6.12 [5.68] x 6.3-8.0 [7.19]), longitud de la tegmina x amplitud, 3.06-3.74 [3.4] x 1.87-2.08 [1.92] (3.06-3.91 [3.49] x 1.87-2.21 [2.0]). Nota: machos $\mathrm{N}=26$, hembras $\mathrm{N}=27$.

Tipos. Holotipo. M 13.3289, REPÚBLICA DOMINICANA, Parque Nacional Armando Bermúdez, Ciénaga de Manabao, provincia La Vega, Jarabacoa, camino a La Virgen, 5-XII2008, cols. Germán de Jesús Adames, E. Gutiérrez; depositado en el MNHNCu. Paratipos. 1M 13.3288, 3H 13.3290-13.3292, 2 ninfas 13.3293-13.3311, con los mismos datos del holotipo; 8M 13.3294-13.3301, 9H 13.3302-13.3310, REPÚBLICA DOMINICANA, Parque Nacional Armando Bermúdez, Ciénaga de Manabao, provincia La Vega, Jarabacoa, camino entre la Ciénaga y los Tablones, bajo corteza de troncos podridos, hojarasca y bromeliáceas, 3-XII-2008, cols. Germán de Jesús Adames, E. Gutiérrez, todos en el MNHNCu, excepto 1M 13.3288, 1H 13.3302 en ANSP y 1M 13.3301 en MNHNSD; 1M 13.3312, 1H 13,3313, 2 ninfas 13.331413.3315, REPÚBLICA DOMINICANA, Parque Nacional Armando Bermúdez, Ciénaga de Manabao, provincia La Vega, Jarabacoa, camino entre los Tablones y el Alto de la Cotorra, en bromeliáceas, 4-XII-2008, cols. Germán de Jesús Adames, E. Gutiérrez, en el MNHNCu; 2M 13.3316-13.3317, 4H 13.3318-13.3321, 1 ninfa 13.3322, REPÚBLICA DOMINICANA, Parque Nacional Armando Bermúdez, Ciénaga de Manabao, provincia La Vega, Jarabacoa, camino entre los Tablones y el Alto de la Cotorra, en bromeliáceas, 5-XII-2008, cols. Germán de Jesús Adames, E. Gutiérrez, en el MNHNCu excepto la H 13.3319 en MNHNSD; 1M 13.3323 MNHNCu, REPÚBLICA DOMINICANA, Parque Nacional Armando Bermúdez, Sector N Antón Sape Malo, dentro de yaguas de palma Manacla y ramas de helechos arborescentes, 8-XII2008, cols. E. Gutiérrez, Florencio A. Peralta, Héctor Andújar; 1M, 1H NHNH, REPÚBLICA DOMINICANA, RD-157, Los Tablones, Parque Nacional Armando Bermúdez, provincia La Vega, $19^{0} 03.308^{\prime} \mathrm{N}, 70^{\circ}$ 53.049'W, 1,279 m, 23-VII-2003, D. Pérez, R. Bastardo, B. Hierro (night); 2H NHNH, REPÚBLICA DOMINICANA, RD-049, Los Tablones, Parque Nacional Armando Bermúdez, provincia La Vega, $1^{\circ} 03.308^{\prime} \mathrm{N}, 70^{\circ} 53.049^{\prime} \mathrm{W}, 19-\mathrm{VII}-2002$, D. Pérez, B. Hierro, R. Bastardo, S. Medrano, H. Takizagua; 1M NMNH, REPÚBLICA DOMINICANA, RD-250, La Ciénaga-Los Tablones, Parque Nacional Armando Bermúdez, provincia La Vega, $19^{\circ} 04.044^{\prime} \mathrm{N}, 70^{\circ} 51.789^{\prime} \mathrm{W}, 1,100-1270$ m, 29-VI-2004, D. Pérez (d); 1H FSCA, REPÚBLICA DOMINICANA, Ciénaga de Manabao, provincia La Vega, Park Hdqt. 3-5-VII-1999, $3000 \mathrm{ft}$. Elev., R. E. Woodruff, at night; 2M 13.2969-13.2970 MNHNCu, REPÚBLICA DOMINICANA, NE Parque Nacional Armando Bermúdez, loma Alto de la Bandera (1470 m), Jánico, provincia Santiago, 11-III-1999 (pluvisilva montana), col. L. F. Armas; 1M, 1H NMNH, REPÚBLICA DOMINICANA, PNJAB, Cerro Prieto, Jánico, provincia Santiago, 10-14-III-1999, col. R. Bastardo; 1H 13.2971 MNHNCu, REPÚBLICA DOMINICANA, NE Parque Nacional Armando Bermúdez (1405 m), 70 50' 27.9'”W, $19^{0} 07^{\circ} 45^{\prime} \mathrm{N}$, Jánico, provincia Santiago, 13-III-1999 (bajo tronco podrido pluvisilva), col. L. F. Armas; 1M MNHNCu, REPÚBLICA DOMINICANA, NE Parque Nacional Armando Bermúdez, $19^{0} 07^{\prime}$ 5.6”N, 71 00' 17.9' W, 1190 m, Sección Rincón de Piedra de San José de las Matas, provincia Santiago, 8-IV-1999 (bajo piedras y hojarasca), col. L. F. Armas; 4M ANSP, REPÚBLICA DOMINICANA, Loma Rucilla y mts. N, June'38, 5-8000 ft., Darlington; 1M, 1H MNHNSD, REPÚBLICA DOMINICANA, Pinar Parejo, Valle Nuevo, Estación VIII, 10-VII-1998, D. Veloz, S. Navarro; 1M, 1H ANSP, REPÚBLICA DOMINICANA, Valle Nuevo, SE Constanza, Aug.'38, c. 7000 ft., Darlington; 1H ANSP, REPÚBLICA DOMINICANA, Constanza to Valle Nuevo, Aug.'38, 3-7000 ft., Darlington; 1H ANSP, REPÚBLICA DOMINICANA, Loma Vieja, S Constanza, Aug.' 38, c. $6000 \mathrm{ft}$., Darlington.

Comentario. Eurycotis adamesi sp. nov. fue documentada de día refugiada bajo corteza de troncos podridos, en bromeliáceas, dentro de ramas de helechos arborescentes, bajo piedras y 
hojarascas (Fig. 4A), en el interior de yaguas (vainas) de palma manacla (Prestoea montana) muertas en el suelo, y bajo las mismas de palmas en pie (Fig. 4B). La especie se distribuye ampliamente en la Cordillera Central a diferentes elevaciones, en localidades del Parque Nacional Armando Bermúdez, en Valle Nuevo y cercanías de Constanza, Pinar Parejo, Loma Rucilla, entre otras localidades como se listan en el acápite Tipos. Algunos especímenes estudiados de diversas poblaciones muestran pequeñas diferencias en el patrón de coloración del abdomen; sin embargo, no existen caracteres morfológicos suficientes para considerarlos especies diferentes. Con mayores esfuerzos de recolectas y la aplicación de métodos modernos se podrá corroborar o cambiar el actual criterio sobre el alcance y distribución de la especie.

\section{Eurycotis neiba sp. nov.}

Figura 5, A-L

Diagnosis. Eurycotis neiba sp. nov., comparte el carácter tibia III ensanchada e hinchada solo con $E$. rothi y E. adamesi sp. nov. de República Dominicana. De E. rothi se diferencia en presentar la tibia III ligeramente menos ensanchada e hinchada (Fig. 5, F-H) (E. rothi, Fig. 7, E-G en Gutiérrez, 2013), y de E. adamesi sp. nov., en presentar la tibia III ligeramente más ensanchada e hinchada (E. adamesi sp. nov., Fig. 3, F-H). De ambas especies, E. neiba sp. nov. difiere en la forma del esclerito R2 del falómero derecho del macho (Fig. 5L) (E. rothi, Fig. 7K en Gutiérrez, 2013; E. adamesi sp. nov., Fig. 3L).

Diagnosis. Eurycotis neiba sp. nov., shares the character widened and inflated tibia III only with $E$. rothi and $E$. adamesi sp. nov. from Dominican Republic. From E. rothi it differs in having tibia III slightly less widened and inflated (Fig. 5, F-H) (E. rothi, Fig. 7, E-G in Gutiérrez, 2013), and from $E$. adamesi sp. nov., in having tibia III slightly more widened and inflated (E. adamesi sp. nov., Fig. 3, F-H). From both species E. neiba sp. nov., differs in the shape of the sclerite R2 of the male right phallomere (Fig. 5L) (E. rothi, Fig. 7K in Gutiérrez, 2013; E. adamesi sp. nov., Fig. 3L).

Descripción. Macho holotipo: $19.2 \mathrm{~mm}$. Superficie dorsal del cuerpo lisa con puntuaciones finas, ligeramente más marcadas en las tegminas, terguitos 5-7 y placa supra-anal. Cabeza ligeramente expuesta; distancia entre las bases de las antenas ligeramente menor que el espacio interocular (Fig. 5C). Pronoto subtriangular con el margen caudal sinuoso, ligeramente convexo en su porción media (Fig. 5D). Tegminas laterales, escamiformes, más anchas en la base, se estrechan hacia el ápice, margen apical ligeramente redondeado, no sobrepasan el margen posterior del mesonoto (Fig. 5, A y E). Alas posteriores ausentes. Fémur I con hilera de espinas robustas en el margen anterior que terminan en tres espinas apicales más largas y engrosadas (Tipo $\mathrm{A}_{3}$ ); fémures II y III con espina genicular; tibia III (caudal) con sus caras caudal y cefálica ensanchadas (Fig. 5, F y H), e inflada (Fig. 5G) en su porción media, sin modificación; pulvilos sobre los cuatro tarsómeros proximales; uñas tarsales simétricas, simples, arolio desarrollado. Abdomen con ángulos caudo-laterales del séptimo terguito abdominal simples (no proyectados), sin lamelas. Placa supra-anal con invaginación media en el margen caudal en forma de "V" invertida, márgenes laterales sinuosos; ángulos látero-caudales redondeados (Fig. 5I); cercos puntiagudos (el derecho roto en el holotipo) sobrepasan la placa supra-anal. Placa subgenital simétrica, margen caudal convexo, redondeado; estilos simples, largos, ligeramente recurvados y afinados en el ápice (Fig. 5J). Genitales con el esclerito R2 del falómero derecho como en la Fig. 5L. Color: superficie dorsal (Fig. 5A) bicolor; negro que contrasta con dos bandas anchas amarillo-pálido a naranja que corren a ambos lados del cuerpo y comienzan a nivel de los ángulos ántero-laterales del pronoto hasta los ángulos látero-caudales, continúa a nivel de las tegminas (bicolores, mayormente amarillo naranja pálido excepto banda castaño pálido en el margen interno, Fig. 5E), el metanoto y se hacen intermitentes y reducidas en los terguitos 2-4 

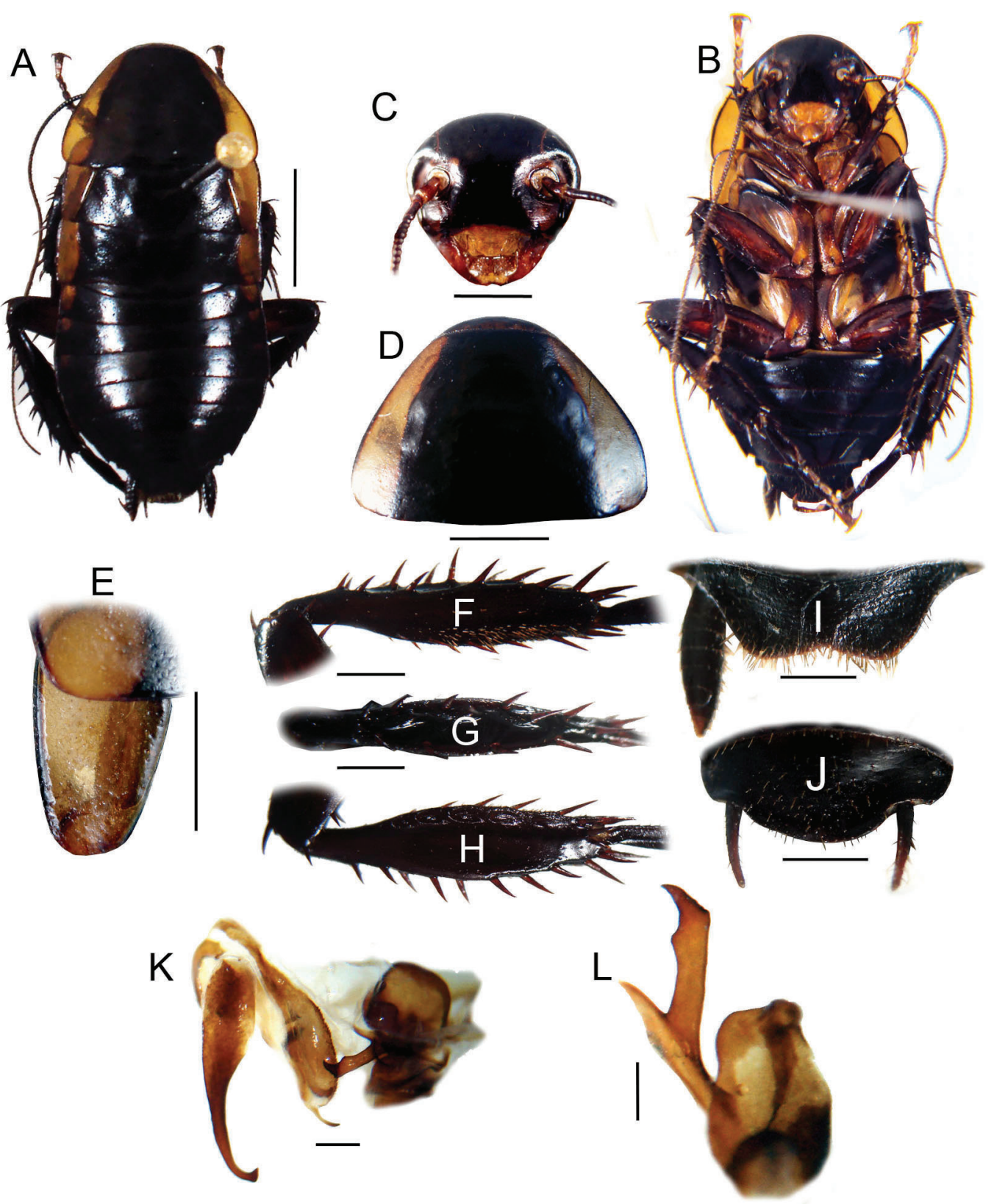

Figura 5, A-L. Eurycotis neiba sp. nov., holotipo macho del MNHNCu. A, aspecto dorsal del cuerpo. B, cuerpo (ventral). C, cabeza (frontal). D, pronoto (dorsal). E, tegmina izquierda (dorsal). F, tibia III derecha (cara caudal). G, ídem anterior (margen dorsal). H, tibia III derecha (cara cefálica). I, placa supra-anal y cerco izquierdo (dorsal). J, placa subgenital y estilos (ventral). K, estructuras genitales (dorsal). L, esclerito R2 del falómero derecho (ventral). Escalas: A-B=5.6 mm, C=2.4 mm, D=2.8 mm, E=2 mm, F-H=1.6 $\mathrm{mm}, \mathrm{I}=1 \mathrm{~mm}, \mathrm{~J}=1.3 \mathrm{~mm}, \mathrm{~K}=0.8 \mathrm{~mm}, \mathrm{~L}=0.4 \mathrm{~mm}$. 
hasta formar un punto pequeño tenue a nivel del terguito 4. Superficie ventral (Fig. 5B): cabeza completamente negra con amarillo pálido en clípeo, labro y palpos; mandíbulas y marcas ocelares naranja pálido. Amarillo pálido en algunas zonas de las coxas; castaño rojizo en trocánteres y fémures; tibias y tarsos castaño oscuro a claro en los últimos artejos tarsales; blanco pálido en pulvilos. Abdomen negro.

Etimología. El nombre propuesto hace alusión a la Sierra del mismo nombre.

Medidas (mm). Macho: longitud del cuerpo, 19.2, longitud del pronoto x amplitud, $5.61 \times$ x 8.5, longitud de la tegmina $\mathrm{x}$ amplitud, $3.57 \times 2.04$. Nota: macho $\mathrm{N}=1$.

Tipos. Holotipo. M 13.2974, REPÚBLICA DOMINICANA, Los Bolos (1100 m), Sierra de Neiba, Postrer Río, provincia Independencia, 14-IV-1999, col. L. F. Armas (bajo piedras); depositado en el MNHNCu.

Comentario. La especie se conoce solo a partir del holotipo y de su localidad tipo. La hembra aun es desconocida para la ciencia. Probablemente E. neiba sp. nov., E. adamesi sp. nov., y E. rothi, (táxones muy emparentados morfológicamente) compartieron un antecesor común que propició la especiación actual en la Sierra de Neiba y la Cordillera Central de la Hispaniola.

\section{Eurycotis hierroi sp. nov.}

Figura 6, A-K

Diagnosis. Eurycotis hierroi sp. nov., difiere de las restantes especies bicolores en presentar el margen caudal de la placa subgenital del macho bilobulado, con invaginación media (Fig. 6I), y en la forma del esclerito R2 del falómero derecho del macho (Fig. 6K).

Diagnosis. Eurycotis hierroi sp. nov., differs from the rest of bicolor species in having an invagination in the subgenital plate caudal margin (Fig. 6I), and in the shape of the sclerite R2 of the male right phallomere (Fig. 6K).

Descripción. Macho holotipo: $17.3 \mathrm{~mm}$. Superficie dorsal del cuerpo lisa, lustrosa, con puntuaciones muy finas. Cabeza ligeramente expuesta; distancia entre las bases de las antenas ligeramente menor que el espacio interocular (Fig. 6C). Pronoto subparabólico con el margen caudal ligeramente convexo (Fig. 6D). Tegminas laterales, escamiformes, más anchas en la base, se estrechan hacia el ápice, margen apical redondeado, sobrepasan ligeramente el margen posterior del mesonoto (Fig. 6, A y E). Alas posteriores ausentes. Fémur I con margen anterior con hilera de espinas robustas, que terminan en tres espinas apicales más largas y engrosadas (Tipo $\mathrm{A}_{3}$ ); fémures II y III con espina genicular; tibia III (caudal) con sus caras caudal y cefálica no ensanchadas (Fig. 6F), y no inflada (Fig. 6G); pulvilos sobre los cuatro tarsómeros proximales; uñas tarsales simétricas, simples, arolio desarrollado. Abdomen con ángulos caudo-laterales del séptimo terguito abdominal simples (no proyectados), sin lamelas. Placa supra-anal con el margen caudal cóncavo, márgenes laterales sinuosos; ángulos látero-caudales redondeados (Fig. 6H); cercos puntiagudos sobrepasan la placa supra-anal. Placa subgenital simétrica, margen caudal convexo con invaginación media; estilos simples, cortos (Fig. 6I). Genitales con el esclerito R2 del falómero derecho como en la Fig. 6K. Color: superficie dorsal (Fig. 6A) bicolor; negra (con viso marrón-rojizo) que contrasta con dos bandas anchas amarillo-pálido a naranja que corren a ambos lados del cuerpo y comienzan a nivel de los ángulos ántero-laterales del pronoto hasta los ángulos látero-caudales, continúa a nivel de las tegminas (bicolores, amarillo pálido excepto banda estrecha oscura en la base del margen interno, Fig. 6E), el metanoto y se hacen intermitentes y más estrechas en los terguitos 2-3. Superficie ventral (Fig. 6B): cabeza negra 

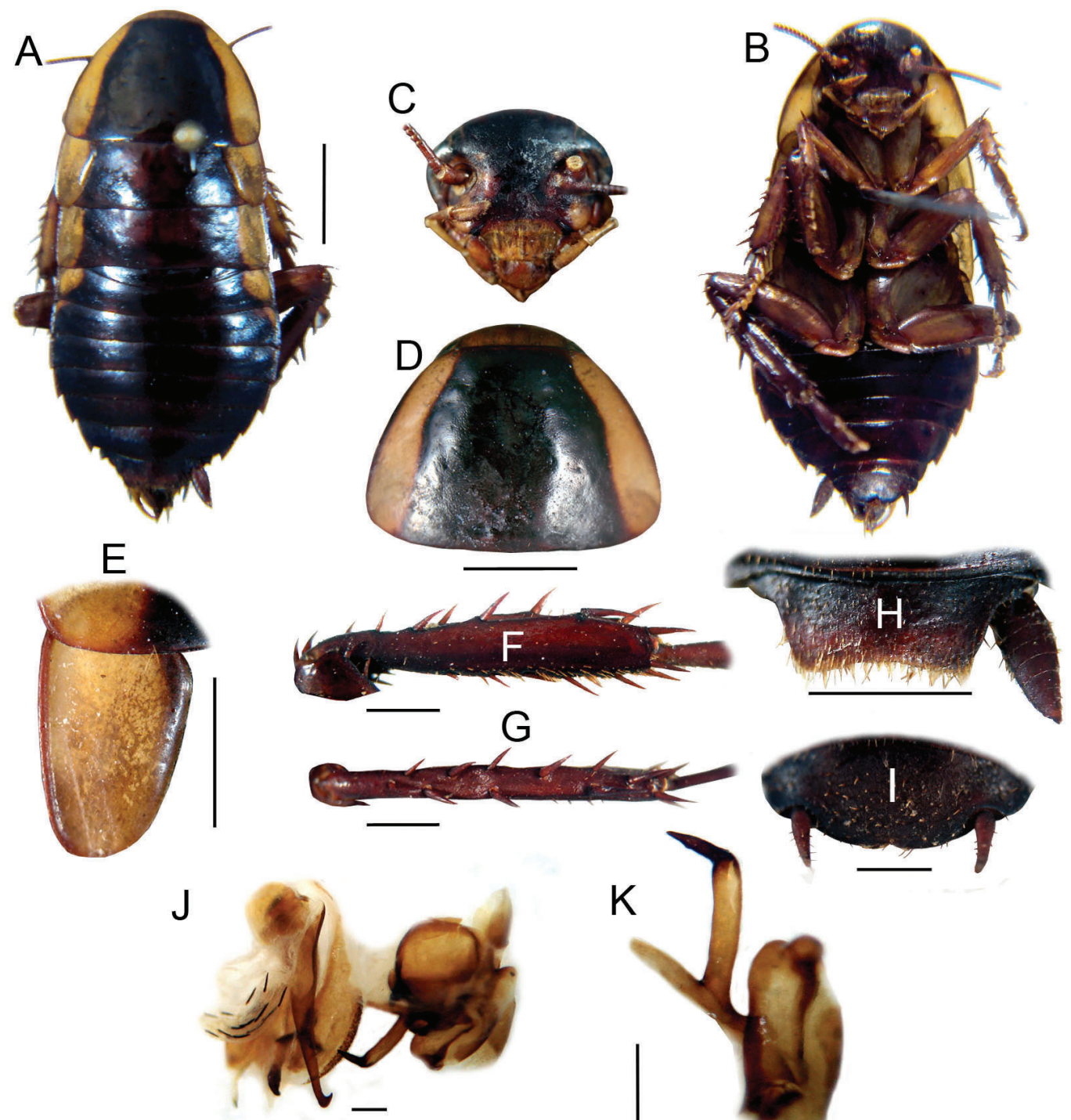

G
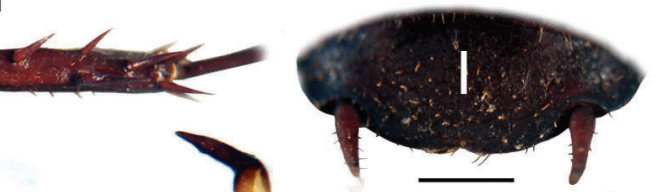

$\mathrm{K}$
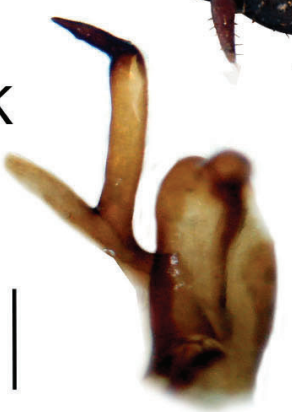

Figura 6, A-K. Eurycotis hierroi sp. nov., holotipo macho del NMNH. A, aspecto dorsal del cuerpo. B, cuerpo (ventral). C, cabeza (frontal). D, pronoto (dorsal). E, tegmina izquierda (dorsal). F, tibia III derecha (cara caudal). G, ídem anterior (margen dorsal). $\mathrm{H}$, placa supra-anal y cerco derecho (dorsal). I, placa subgenital y estilos (ventral). J, estructuras genitales (dorsal). K, esclerito R2 del falómero derecho (ventral). Escalas: $\mathrm{A}-\mathrm{B}=4.7 \mathrm{~mm}, \mathrm{C}=2.8 \mathrm{~mm}, \mathrm{D}=2.38 \mathrm{~mm}, \mathrm{E}=1.87 \mathrm{~mm}, \mathrm{~F}-\mathrm{G}=1.77 \mathrm{~mm}, \mathrm{H}=1.1 \mathrm{~mm}, \mathrm{I}=0.9 \mathrm{~mm}$, $\mathrm{J}-\mathrm{K}=0.4 \mathrm{~mm}$. 
con viso rojizo, con amarillo naranja pálido en clípeo, labro, genas, palpos, mandíbulas; marcas ocelares amarillo pálido (Fig. 6C). Amarillo con viso rojizo a naranja en coxas, trocánteres, fémures, tibias I y II; castaño rojizo en tibias III; amarillo castaño en tarsos, blanco pálido en pulvilos, y abdomen castaño-oscuro rojizo. Hembra: difiere del macho en su mayor talla, en las placas: supra-anal (subtriangular proyectata con invaginación apical), y subgenital (bilobular) características de las hembras del género.

Etimología. El nombre propuesto es un patronímico en honor al colega Brígido Hierro (Dirección de Biodiversidad, Ministerio de Medio Ambiente y Recursos Naturales, Santo Domingo, RD) por su destacada participación en las expediciones que permitieron las recolectas de la mayoría de los táxones nuevos descritos en este estudio.

Medidas (mm). Hembra entre paréntesis: longitud del cuerpo, 17.34 (18.7), longitud del pronoto x amplitud, 4.76 x 6.97 (5.44 x 7.82), longitud de la tegmina x amplitud, 3.23 x 1.87 (3.4 x 2.04). Nota: machos $\mathrm{N}=1$, hembras $\mathrm{N}=1$.

Tipos. Holotipo. M, REPÚBLICA DOMINICANA, RD-148, up 100 m del El Sitio del Agua, bosque nublado N Los Bolos, Sierra de Neiba, provincia Independencia, $18^{0} 39.339^{\prime} \mathrm{N}, 7^{\circ}$ 39.279'W, 1,520 m, 9-VII-2003, D. Pérez, R. Bastardo, B. Hierro (day/night); depositado en el NMNH. Paratipo. 1H, con los mismos datos del holotipo; en el NMNH.

Comentario. Eurycotis hierroi sp. nov., es conocida hasta la fecha de la localidad tipo. Es la segunda especie descrita para la Sierra de Neiba.

\section{Eurycotis quitaespuela sp. nov. \\ Figura 7, A-M}

Diagnosis. Eurycotis quitaespuela sp. nov., comparte los caracteres cuerpo bicolor, tegminas separadas y subrectangulares con E. barahonensis, E. rehni, E. hebardi y E. isabeltorres sp. nov. de República Dominicana. De estos táxones difiere en la forma del esclerito R2 del falómero derecho del macho (Fig. 7K) (E. barahonensis, Fig. 2H; E. rehni, Fig. 6K; E. hebardi, Fig. 5K en Gutiérrez, 2013; E. isabeltorres sp. nov., Fig. 8L).

Diagnosis. Eurycotis quitaespuela sp. nov., shares the characters bicolor body, subrectangular and separated tegmina with E. barahonensis, E. rehni, E. hebardi, and E. isabeltorres sp. nov. from Dominican Republic. From these taxa the new species differs in shape of sclerite R2 of the male right phallomere (Fig. 7K) (E. barahonensis, Fig. 2H; E. rehni, Fig. 6K; E. hebardi, Fig. 5K in Gutiérrez, 2013; E. isabeltorres sp. nov., Fig. 8L).

Descripción. Macho holotipo: $17.25 \mathrm{~mm}$. Superficie dorsal del cuerpo lisa lustrosa, con puntuaciones muy finas esparcidas. Cabeza ligeramente expuesta; distancia entre las bases de las antenas ligeramente menor que el espacio interocular. Pronoto con el margen anterior recto; márgenes caudal y laterales convexos; ángulos látero-posteriores redondeados (Fig. 7C). Tegminas laterales, subrectangulares y más anchas en la base, con el margen interno redondeado, margen distal recto, sobrepasan ligeramente el margen posterior del mesonoto a ambos lados (Fig. 7, A y D). Alas posteriores ausentes. Fémur I con margen anterior con hilera de espinas robustas, que terminan en tres espinas apicales más largas y engrosadas (Tipo $\mathrm{A}_{3}$ ); fémures II y III con espina genicular; tibia caudal ensanchada, aplanada (no inflada) (Fig. 7, E-G) sin modificación definida; pulvilos sobre los cuatro tarsómeros proximales; uñas tarsales simétricas, simples, arolio desarrollado. Abdomen con ángulos caudo-laterales del séptimo terguito abdominal simples (no proyectados), sin lamelas. Placa supra-anal bilobulada con 
invaginación media en el margen caudal en forma de "V" invertida, márgenes laterales sinuosos; ángulos látero-caudales redondeados (Fig. $7 \mathrm{H})$; cercos puntiagudos sobrepasan placa supra-anal. Placa subgenital simétrica, margen caudal convexo; estilos simples, ligeramente recurvados y afinados en el ápice (Fig. 7I). Genitales con el esclerito R2 del falómero derecho como en la Fig. 7K. Color: superficie dorsal bicolor (Fig. 7A); castaño rojizo que contrasta con dos bandas anchas amarillo-pálido que corren a ambos lados del cuerpo; las bandas comienzan a nivel de los ángulos ántero-laterales del pronoto hasta los ángulos láterocaudales, continúan a nivel de las tegminas (amarillas) y el metanoto, las cuales se hacen intermitentes en los terguitos 2-5. Superficie ventral (Fig. 7B): cabeza castaño oscuro, con amarillo en vértice, alrededor de las bases de las antenas en la frente, genas, clípeo, labro, palpos. Amarillo en coxas, trocánteres, fémures; con castaño-claro en tibias y tarsos I-II; castaño rojizo en tibias y tarsos III. Abdomen castaño rojizo con marcas amarillas a ambos lados de los esternitos que coinciden con bandas tergales. Hembra: difiere del macho en su mayor talla, en la placa supra-anal (Fig. 7L) y la placa subgenital (Fig. 7M).

Etimología. El apelativo propuesto es un patronímico compuesto por las palabras de la localidad tipo loma "Quita Espuela", hábitat de la especie.

Medidas (mm). Hembra entre paréntesis, media entre corchetes: longitud del cuerpo, 17.1717.34 [17.25] (20.91), longitud del pronoto x amplitud, 5.44-5.61 [5.52] x 7.82-8.0 [7.91] (6.3 x 8.5), longitud de la tegmina $x$ amplitud, 3.74-4.25 [3.9] x 2.38-2.72 [2.5] (4.42 x 2.38). Nota: machos $\mathrm{N}=2$, hembras $\mathrm{N}=1$.

Tipos. Holotipo. M 13.2354, REPÚBLICA DOMINICANA, Loma Quita Espuela, San Francisco de Macorís, provincia Duarte, 11-II-2002, en la base de hoja de palma viva, col. E. Gutiérrez; depositado en el MNHNCu. Paratipos. 1M 13.2355 MNHNSD, 1H 13.2356 MNHNCu, 1 ninfa 13.2357 $\mathrm{MNHNCu}$, con los mismos datos del holotipo, excepto la $\mathrm{H} 13.2356$ que fue colectada en bromeliácea.

Comentario. La especie es conocida hasta la fecha de la localidad tipo al SE de la Cordillera Septentrional.

Eurycotis isabeltorres sp. nov.

Figura 8, A-L

Diagnosis. Eurycotis isabeltorres sp. nov., comparte los caracteres cuerpo bicolor, tegminas separadas y subrectangulares con E. barahonensis, E. rehni, E. hebardi y E. quitaespuela sp. nov., de República Dominicana. De estos táxones difiere en la forma del esclerito R2 del falómero derecho del macho, Fig. 8L (E. barahonensis, Fig. 2H; E. rehni, Fig. 6K; E. hebardi, Fig. 5K en Gutiérrez, 2013; E. quitaespuela sp. nov., Fig. 7K).

Diagnosis. Eurycotis isabeltorres sp. nov., shares the characters bicolor body, subrectangular and separated tegmina with E. barahonensis, E. rehni, E. hebardi, and E. quitaespuela sp. nov. from Dominican Republic. From these taxa the new species differs in shape of sclerite R2 of the male right phallomere, Fig. 8L (E. barahonensis, Fig. 2H; E. rehni, Fig. 6K; E. hebardi, Fig. 5K in Gutiérrez, 2013; E. quitaespuela sp. nov., Fig. 7K).

Descripción. Macho holotipo: $19.38 \mathrm{~mm}$. Superficie dorsal del cuerpo lisa lustrosa, con puntuaciones muy finas esparcidas. Cabeza ligeramente expuesta; distancia entre las bases de las antenas ligeramente menor que el espacio interocular (Fig. 8C). Pronoto más largo que ancho, con el margen anterior ligeramente cóncavo; margen caudal truncado, ligeramente sinuoso; márgenes 


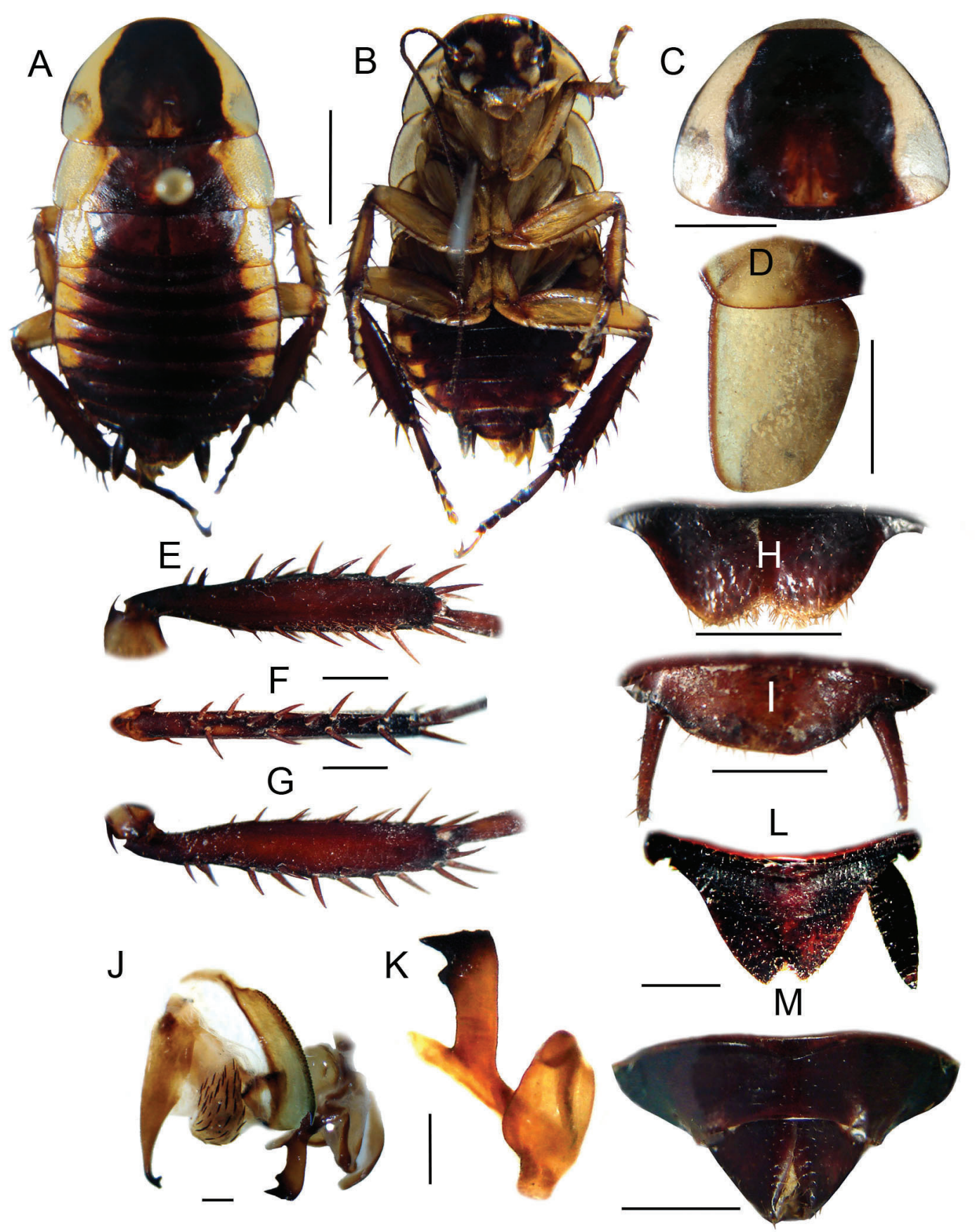

Figura 7, A-M. Eurycotis quitaespuela sp. nov. A-K, holotipo macho del MNHNCu. A, aspecto dorsal del cuerpo. B, cuerpo (ventral). C, pronoto (dorsal). D, tegmina izquierda (dorsal). E, tibia III derecha (cara caudal). F, ídem anterior (margen dorsal). G, tibia III derecha (cara cefálica). H, placa supra-anal (dorsal). I, placa subgenital y estilos (ventral). J, estructuras genitales (dorsal). K, esclerito R2 del falómero derecho (ventral). L-M, hembra. L, placa supra-anal y cerco derecho (dorsal). M, placa subgenital (ventral). Escalas: A-B=5.5 mm, C=2.72 mm, D=2.5 mm, E-G=2 mm, H=2.2 mm, I=1.2 mm, J-K=0.5 mm. 

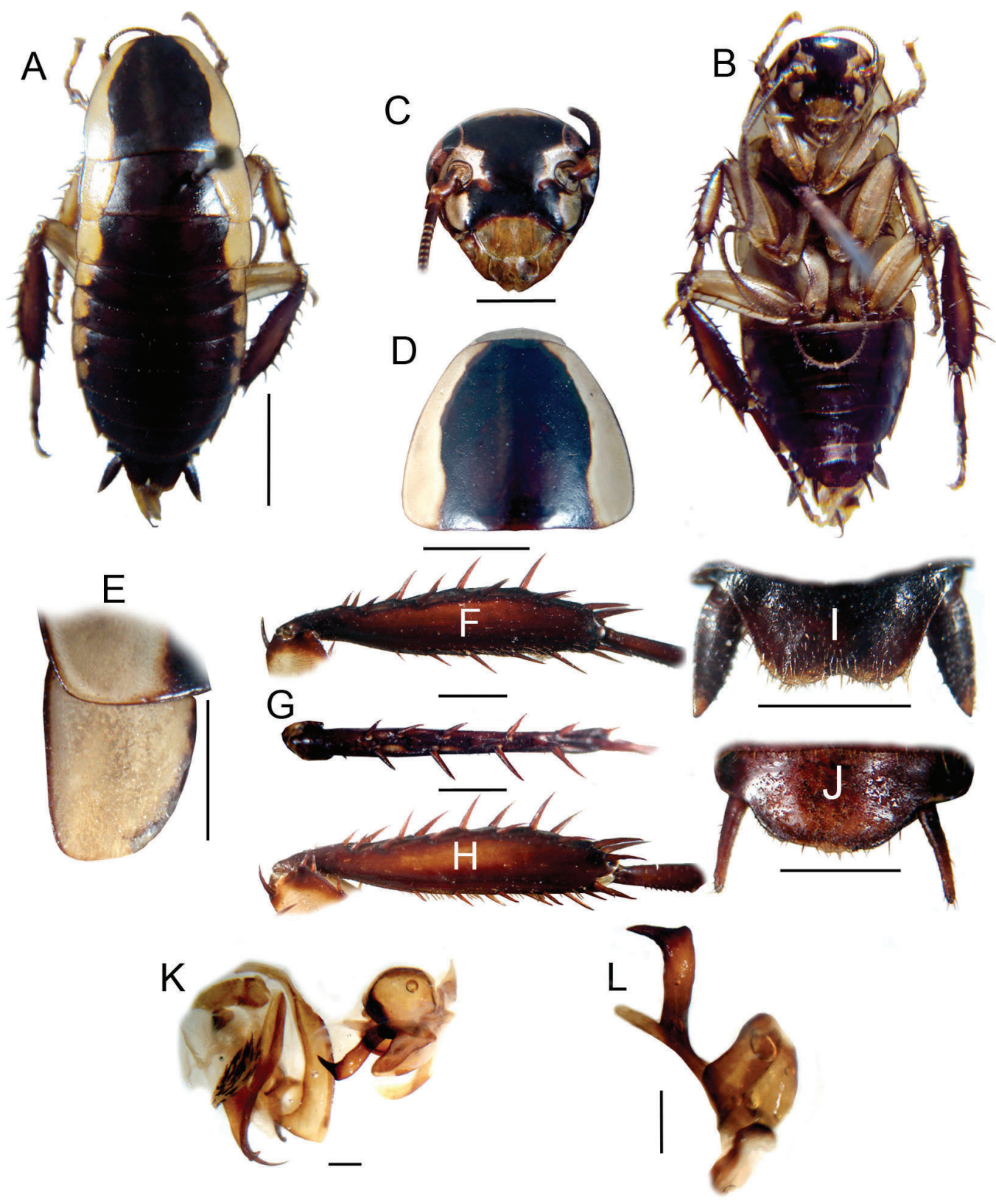

Figura 8, A-L. Eurycotis isabeltorres sp. nov., holotipo macho del MNHNCu. A, aspecto dorsal del cuerpo. B, cuerpo (ventral). C, cabeza (frontal). D, pronoto (dorsal). E, tegmina izquierda (dorsal). F, tibia III derecha (cara caudal). G, ídem anterior (margen dorsal). H, tibia III izquierda (cara cefálica). I, placa supra-anal y cercos (dorsal). J, placa subgenital y estilos (ventral). K, estructuras genitales (dorsal). L, esclerito R2 del falómero derecho (ventral). Escalas: $A-B=5.4 \mathrm{~mm}, \mathrm{C}=2.2 \mathrm{~mm}, \mathrm{D}=2.72 \mathrm{~mm}, \mathrm{E}=2.4 \mathrm{~mm}$, $\mathrm{F}-\mathrm{H}=1.8 \mathrm{~mm}, \mathrm{I}=1.9 \mathrm{~mm}, \mathrm{~J}=1.2 \mathrm{~mm}, \mathrm{~K}-\mathrm{L}=0.5 \mathrm{~mm}$. 
laterales convexos; ángulos látero-posteriores redondeados (Fig. 8D). Tegminas laterales, subrectangulares, más anchas en la base, con el margen interno redondeado, margen distal recto, sobrepasan ligeramente el margen posterior del mesonoto a ambos lados (Fig. 8, A y E). Alas posteriores ausentes. Fémur I con margen anterior con hilera de espinas robustas, que terminan en tres espinas apicales más largas y engrosadas (Tipo $\mathrm{A}_{3}$ ); fémures II y III con espina genicular; tibia caudal ensanchada, aplanada (no inflada) (Fig. 8, F-H) sin modificación definida; pulvilos sobre los cuatro tarsómeros proximales; uñas tarsales simétricas, simples, arolio desarrollado. Abdomen con ángulos caudo-laterales del séptimo terguito abdominal simples (no proyectados), sin lamelas. Placa supra-anal bilobulada con invaginación media en el margen caudal en forma de "U" invertida, márgenes laterales sinuosos; ángulos látero-caudales redondeados (Fig. 8I); cercos puntiagudos sobrepasan la placa supra-anal. Placa subgenital simétrica, margen caudal convexo; estilos simples, ligeramente recurvados y afinados en el ápice (Fig. 8J). Genitales con el esclerito R2 del falómero derecho como en la Fig. (8L). Color: superficie dorsal bicolor (Fig. 8A); castaño oscuro rojizo a negro que contrasta con dos bandas anchas amarillo-pálido que corren a ambos lados del cuerpo; las bandas comienzan a nivel de los ángulos ántero-laterales del pronoto hasta los ángulos látero-caudales, continúa a nivel de las tegminas (amarillas), el metanoto y se hacen intermitentes en los terguitos 2-5. Superficie ventral (Fig. 8B): cabeza castaño oscuro a negro, con blanco pálido en el vértice, alrededor de las bases de las antenas en la frente y genas; amarillo en clípeo, labro, palpos. Amarillo en coxas, trocánteres, fémures, tibias y tarsos I-II; castaño rojizo en tibias y tarsos III; blanco pálido en pulvilos y arolios. Abdomen castaño rojizo con marcas amarillas a ambos lados de los esternitos que coinciden con bandas tergales. Hembra: difiere del macho en las placas: supra-anal (subtriangular proyectada con invaginación apical), y subgenital (bilobular) características de las hembras del género.

Etimología. El nombre propuesto es un patronímico compuesto por dos de las palabras que conforman la localidad tipo loma "Isabel de Torres", hábitat de la especie.

Medidas (mm). Hembra entre paréntesis, media entre corchetes: longitud del cuerpo, 18.8719.90 [19.38] (15.13-20.91 [18.16]), longitud del pronoto x amplitud, 5.44-5.44 [5.44] x 6.637.65 [7.14] (5.44-6.3 [5.64] x 7.14-8.16 [7.45]), longitud de la tegmina $x$ amplitud, 3.57-3.74 [3.65] x 2.38-2.55 [2.46] (3.57-4.25 [3.74] x 2.38-2.72 [2.49]). Nota: machos $\mathrm{N}=2$, hembras $\mathrm{N}=6$.

Tipos. Holotipo. M 13.3344, REPÚBLICA DOMINICANA, Loma Isabel de Torres, Puerto Plata, 13-XII-2007, cols. E. Gutiérrez, A. Pérez-Asso, en bromeliáceas; depositado en el

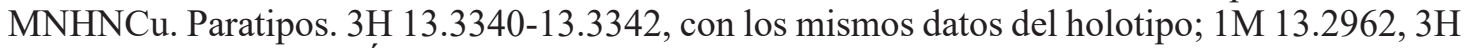
13.2963-13.2965, REPÚBLICA DOMINICANA, Pico Isabel de Torres (790 m), Puerto Plata, 26-II-1999, col. L. F. Armas, en troncos podridos y yaguas. Todos en el MNHNCu, excepto la H 13.3342 en el MNHNSD.

Comentario. La especie es conocida hasta la fecha de la localidad tipo.

Eurycotis mercedes sp. nov.

Figuras 9, A-G

Diagnosis. Eurycotis mercedes sp. nov., comparte con las especies Eurycotis cribosa (Cuba), E. jaragua y E. ruthae (República Dominicana) los caracteres cuerpo robusto, globoso con puntuaciones y tegminas separadas. La especie nueva se diferencia en presentar tegminas subrectangulares (Fig. 9E) (subcuadradas en: E. cribosa y E. jaragua; subovaladas en: E. ruthae), y en tener el pronoto oscuro con banda marginal ántero-lateral clara contrastante (Fig. 9D) (oscuro en: E. cribosa y E. ruthae; claro con manchas oscuras en el disco en: E. jaragua). 


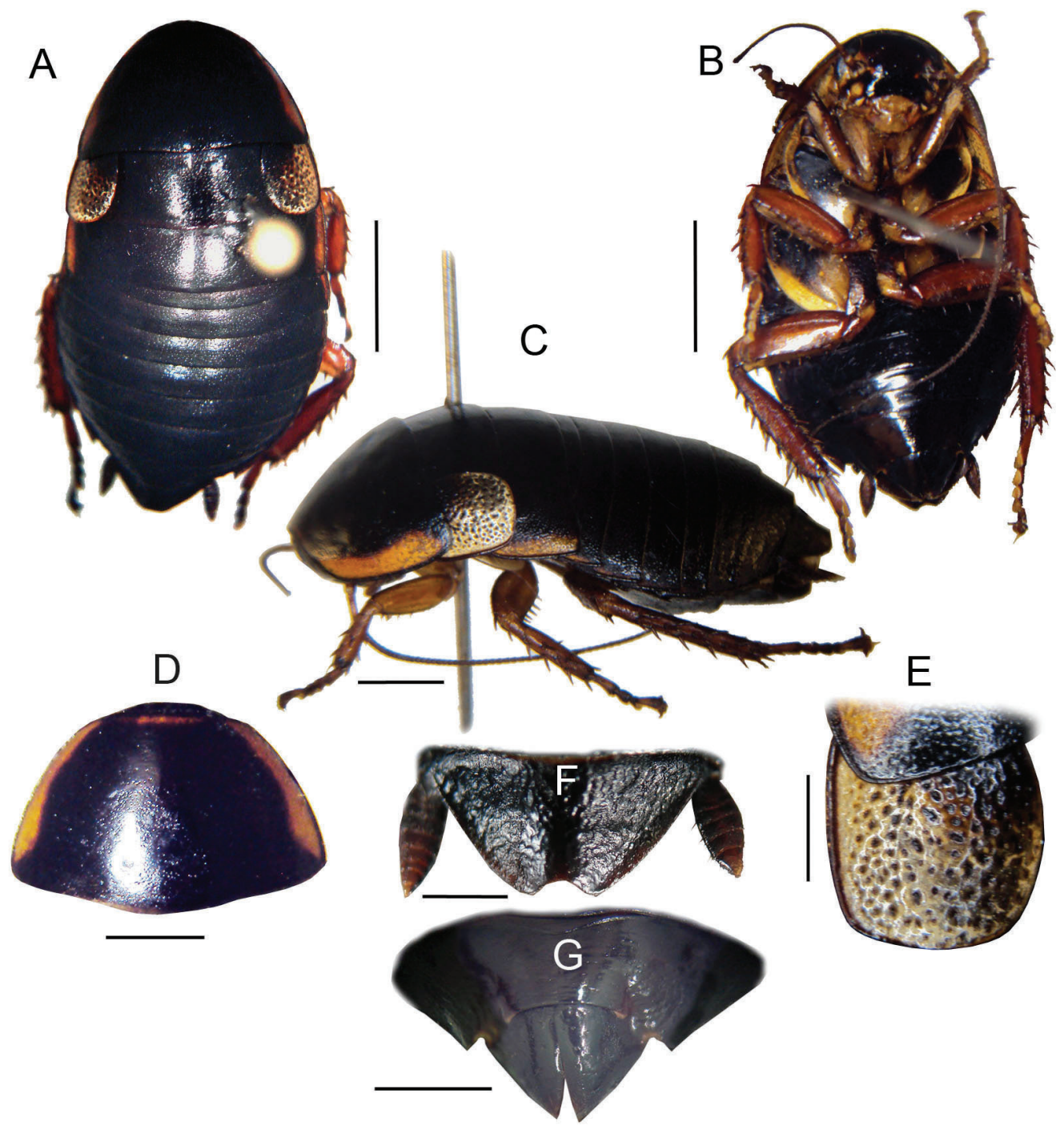

Figura 9, A-G. Eurycotis mercedes sp. nov. A-M, holotipo hembra del MNHNCu. A, aspecto dorsal del cuerpo. B, cuerpo (ventral). $\mathrm{C}$, cuerpo (lateral). D, pronoto (dorsal). E, tegmina izquierda (dorsal). F, placa supra-anal y cercos (dorsal). G, placa subgenital (ventral). Escalas: $A-B=5.1 \mathrm{~mm}, C=2.72 \mathrm{~mm}, \mathrm{D}=2.55 \mathrm{~mm}, \mathrm{E}=1.36 \mathrm{~mm}, \mathrm{~F}=1 \mathrm{~mm}, \mathrm{G}=1.2 \mathrm{~mm}$. 
Diagnosis. Eurycotis mercedes sp. nov., shares the characters body robust, globose, punctate, and separated tegmina with Eurycotis cribosa (Cuba), E. jaragua and E. ruthae (Dominican Republic). The new species differs in having subrectangular tegmina (Fig. 9E) (subquadrate in: E. cribosa and E. jaragua; subovate in: E. ruthae), and in having the pronotum with a contrasting anterolateral marginal band (Fig. 9D) (dark in: E. cribosa y E. ruthae; light with dark spots on the disk in: E. jaragua).

Descripción. Hembra holotipo: especie globosa, coriácea, con apariencia de coleóptero. Superficie dorsal del cuerpo cubierta por puntuaciones finas y unidas, más separadas, amplias y profundas en las tegminas, rugosas en los últimos terguitos a partir del sexto (Fig. 9A). Cabeza ligeramente expuesta, distancia entre las bases de las antenas ligeramente menor que el espacio interocular; manchas ocelares llamativas. Pronoto con los márgenes ántero-laterales curvos y el caudal sinuoso, proyectado en su porción media (Fig. 9D). Tegminas laterales, subrectangulares, con los ángulos póstero-laterales internos redondeados, sobrepasan ligeramente el mesonoto (Fig. 9E). Alas posteriores ausentes. Fémur I con margen anterior con hilera de espinas robustas, que terminan en tres espinas apicales más largas y engrosadas (Tipo $\mathrm{A}_{3}$ ); tibia posterior no ensanchada, ni inflada; pulvilos sobre los cuatro tarsómeros proximales; uñas tarsales simétricas, simples, arolio desarrollado. Abdomen con ángulos caudo-laterales del séptimo terguito abdominal simples (no proyectados), sin lamelas. Placa supra-anal subtriangular; margen caudal con invaginación media en forma de "U" invertida abierta; cercos puntiagudos, no sobrepasan la placa supra-anal (Fig. 9F). Placa subgenital bilobulada, característica de las hembras del género (Fig. 9G). Color: superficie dorsal del cuerpo (Fig. 9A) negra, contrasta con banda naranja ántero-lateral en el pronoto, más estrecha e intermitente sobre el margen anterior, se ensancha paulatinamente a los lados de los márgenes laterales (Fig. 9D), continúa a ambos lados del metanoto y se estrecha a nivel de los márgenes laterales en los terguitos 2-4 (Fig. 9C). Tegmina amarillo pálido cubierta por puntos y manchas irregulares negras (Fig. 9E). Superficie ventral del cuerpo (Fig. 9B) negra, contrasta con manchas ocelares naranja; genas y bases de las mandíbulas blancas; clípeo y labro pálidos; coxas con margen externo fileteado de amarillo-pálido; fémures, tibias y tarsos castaño-naranja.

Etimología. El nombre propuesto alude a la localidad tipo.

Medidas (mm). Hembra: longitud del cuerpo, 17.0, longitud del pronoto x amplitud, 5.1 x 7.82, longitud de la tegmina $\mathrm{x}$ amplitud, $3.23 \times 2.72$. Nota: hembra $\mathrm{N}=1$.

Tipos. Holotipo. H 13.2345, REPÚBLICA DOMINICANA, Las Mercedes, provincia Pedernales, 5-II-2002; depositado en el MNHNCu.

Comentario. Eurycotis mercedes sp. nov., se conoce a partir de un solo ejemplar hembra recolectado de día, refugiado en una planta bromeliácea en la localidad tipo en febrero de 2002. Infructuosos esfuerzos de recolectas posteriores en la localidad tipo, se realizaron durante días y noches en noviembre de 2003. Nos llamó la atención el decline de la población de bromeliáceas de un año al otro, aparentemente debido al uso de las mismas como fuente de alimento y agua para el ganado, según manifestaron varios campesinos encuestados al respecto. Nos alienta pensar que tal vez "Las Mercedes", área bastante degradada por el hombre, sea límite de distribución de la especie, y aún habite otras áreas de la Sierra de Bahoruco. Se requiere realizar futuros esfuerzos de recolectas para lograr conocer la distribución de la especie en la isla, así como el macho de este taxon, aún desconocido. 


\section{AGRADECIMIENTOS}

Sinceros agradecimientos a todas las personas e instituciones dominicanas y extranjeras mencionadas en la primera parte de este estudio (Gutiérrez, 2013) que posibilitaron esta contribución. A todos los colectores de las especies aquí descritas. Por el préstamo de ejemplares a Daniel E. Pérez Gelabert (NMNH), R. E. Woodruff (FSCA) y Carlos Suriel (MNHNSD). Al colega Luis M. Díaz (MNHNCu), por su gentileza de transportar en dos ocasiones desde el extranjero especímenes en préstamo relevantes para esta investigación. Eterno agradecimiento a Gilberto Silva (MNHNCu) por estar siempre dispuesto a revisar mis artículos, y en especial, por la excelente revisión crítica, como de costumbre, del presente manuscrito. A los revisores del grupo editorial de Novitates Caribaea por sus oportunas sugerencias. Agradezco al colega Giraldo Alayón (MNHNCu) su disponibilidad, siempre presto atender mis consultas. Mi gratitud a Daniel E. Pérez-Gelabert y Karolyn Darrow (NMNH) por la gestión, obtención y envío de las excelentes fotografías del holotipo y alotipo de Eurycotis tibialis que ilustran este trabajo. Agradecimientos muy especiales a la señora Nereida Negrín por su valiosa ayuda al autor en su recuperación de una enfermedad eventual, y por haberlo transportado junto a sus colegas a "Loma Quita Espuela" en febrero de 2002, lo cual posibilitó el descubrimiento de una especie nueva aquí descrita. A "Mili" administradora de la reserva Loma Isabel de Torres por sus certeras orientaciones y brindar refugio en su casa durante la Tormenta tropical Olga en diciembre de 2007, que permitió el éxito del trabajo de campo. A Germán de Jesús Adames y Florencio Antonio Peralta (Flor) supervisores de la División Norte del Parque Nacional Armando Bermúdez por su asistencia en el trabajo de campo en diciembre de 2008. Al "grant" de la Iniciativa Taxonómica Global (GTI) del punto focal Belga de Bruselas, por financiar el estudio de colecciones de los tipos del género Eurycotis en instituciones europeas. "Grants" de la ANSP y del Museo Americano de Historia Natural de Nueva York (AMNH) financiaron estudios de colecciones en instituciones norteamericanas. A IDEA WILD por su donativo en equipos. Este estudio se desarrolla en el marco del proyecto "Historia natural de algunos grupos de la fauna del Caribe: su exhibición y divulgación” del MNHNCu.

\section{LITERATURA CITADA}

Gurney, A. B. 1942. Studies in Cuban Blattidae (Orthoptera). Bulletin of the Museum of Comparative Zoology 89(2):11-60.

Gutiérrez, E. 2013. El género Eurycotis (Dictyoptera: Blattaria: Blattidae: Polyzosteriinae). 1. Especies de la Hispaniola. Primera parte: seis especies nuevas. Novitates Caribaea 6:13-35.

Gutiérrez, E. y D. E. Pérez-Gelabert. 2000. Annotated Checklist of Hispaniolan Cockroaches. Transactions of the American Entomological Society 126(3+4): 423-445.

Hebard, M. 1916. Two new dark-colored species of the genus Eurycotis (Orthoptera, Blattidae). Entomological News 27: 263-266.

McKittrick, F. A. 1964. Evolutionary studies of cockroaches. Cornell Experiment Station Memoir 389: 1-197.

Perez-Gelabert, D. E. 2008. Arthropods of Hispaniola (Dominican Republic and Haiti): A checklist and bibliography. Zootaxa 1831: 1-530.

Roth, L. M. 2003. Systematics and phylogeny of cockroaches (Dictyoptera: Blattaria). Oriental Insects 37: 1-186.

[Recibido: 10 de agosto, 2014. Aceptado para publicación: 12 de septiembre, 2014] 